\title{
Me manus una capit: Von kleinen Büchern und ihren Lesern in Martials Epigrammen
}

\section{Ein Spanier in Rom oder literarische Fankultur}

\author{
„Hast Du nie die Geschichte von dem Gaditaner gelesen, der sich, durch den glorreichen Namen \\ des Titus Livius angezogen, vom Ende der Welt aufmachte, um ihn persönlich in Augenschein zu \\ nehmen, und dann gleich, als er ihn gesehen hatte, wieder davonging?“1
}

Dieses adhortative exemplum, mit dem der jüngere Plinius seinen Freund Nepos dazu bewegen will, sich zu den öffentlichen Auftritten des Philosophen Isaeus nach Rom zu begeben, ist ein bemerkenswertes Dokument der Faszination, die zeitgenössische Literaten bereits in der Antike auf ihre Leser auszuüben vermögen, so dass man nicht zuletzt angesichts der enormen Entfernung von Cádiz nach Rom versucht wäre, von einer Art Fan-Kultur zu sprechen, deren Wertschätzung sich in Meilen quantifizieren ließe. ${ }^{2}$

So kurios uns diese Episode erscheinen mag, so illustriert sie doch einige Aspekte, die auch für die Betrachtung der Beziehung von Martial zu seinen Lesern von einiger Relevanz sind. Insbesondere die affektive Bindung des Lesers an den Autor und die Akzentuierung der im Rahmen einer breit entwickelten Buchkultur der literarischen Kommunikation inhärenten Spannung von Nähe und Distanz zwischen Autor und Leser ist hier namhaft zu machen.

\section{Literarische Öffentlichkeit und die Figur des anonymen Lesers}

Dieses Spannungsverhältnis gilt insbesondere angesichts der durchaus massiven Entwicklungen und Veränderungen, die sich im Lauf der frühen Kaiserzeit im Bereich des Literaturbetriebs vollziehen. Wir können eine ausgeprägte Diversifizierung der Medien literarischer Kommunikation beobachten, die mit einer deutlich erhöhten Präsenz literarischer Texte im öffentlichen Raum verbunden ist. Hier ist ebenso die Entstehung öffentlicher Bibliotheken und literarischer Agone hinzuweisen, wie auf

1 Plin. epist. 2,3,8: Numquamne legisti Gaditanum quendam Titi Livi nomine gloriaque commotum ad visendum eum ab ultimo terrarum orbe venisse statimque, ut viderat, abisse?

2 Die Route des antiken Reisenden auf dieser Strecke lässt sich im Übrigen auf dem auf den sog. Vicarello-Bechern inschriftlich (CIL 11,3281-3284) erhaltenen Reiseplan en détail mit allen Stationen auf seiner gesamten Länge von 1895 Meilen (2364 Kilometer) nachvollziehen. 
die Ausbreitung des Rezitationswesens, das von Vortrag beim Symposion über Autorenlesungen in eigens für diesen Zweck gedachten Auditorien bis hin zur spontanen Rezitation auf öffentlichen Plätzen reicht. ${ }^{3}$ Gerade in diesem Bereich können wir beobachten, dass ein zentraler Bestandteil der literarischen Kommunikation eine Kultur der Aufmerksamkeit oder besser der Aufmerksamkeitsgewinnung ist, mit der sich Autoren im Kontext von Literaturförderung zu etablieren trachten. In dieser Kultur der Aufmerksamkeit und der öffentlichkeitswirksamen Inszenierung von Literatur und Literaten spielt natürlich auch ein sich dynamisch entwickelnder Buchhandel samt seiner medialen und buchtechnischen Innovationen eine wichtige Rolle. Die Konstruktion eines auktorialen Images und seine Propagierung sowie Strategien der Gewinnung und Bindung von Lesern werden zu einem unverzichtbaren Bestandteil des literarischen Geschäfts.

Diese Diversifizierung betrifft natürlich auch die Rezeptionssituationen und Rezipienten von Literatur. Neben Elementen der traditionellen face to face-Kommunikation mit definierten Adressaten sowie räumlich und personell beschränkten Rezeptionssituationen (z. B. convivium) wie sie gerade im Kontext der Literaturpatronage und des Klientelwesens nach wie vor von Bedeutung ist, etablieren sich Publika, die weder sozial noch lokal eindeutig zu bestimmen sind. Signifikantestes Merkmal für die hier skizzierten Entwicklungen ist das Erscheinen der Figur eines anonymen Lesers, die nach ihrem ersten prominenten Auftritt bei Ovid gerade in Martials Werk omnipräsent ist und Gegenstand intensiver Reflexion ist. ${ }^{4}$ Insbesondere die Imagination und Auslotung unterschiedlicher Rezeptionsdispositive lässt sich in den von Martial vorgestellten anonymen Rezipientenfiguren beobachten. Wollen wir dies auf das oben angesprochene Verhältnis von Nähe und Distanz beziehen, können wir in der Tat feststellen, dass sich die Reichweite literarischer Kommunikation erhöht, was natürlich zugleich mit einer erhöhten Distanz zwischen Autor und Publikum einhergeht. Dies hat zur Folge, dass die Aufmerksamkeit des Lesers und die Akzeptanz des Autors nicht nur bei einigen wenigen Adressaten, sondern in einem kollektiv gedachten Lesepublikum zum Signet des literarischen Erfolges wird. ${ }^{5}$ Der Leser und seine Reaktion werden damit notwendigerweise zu einem zentralen Thema: So ist bei Martial die Rede vom lector studiosus, ${ }^{6}$ dem enthusiastischen Leser, der nach jedem neuen Martialtext giert und von der Lektüre nicht genug bekommen kann, dem lector gulosus oder delicatus, ${ }^{7}$

$3 \mathrm{Zu}$ diesem Komplex der dynamischen Entwicklung des römischen literarischen Markts und seiner Medien siehe u. a. Kleberg 1967; Binder 1995; Vössing 1997, Fantham 1998; Krasser 1999; Krasser 2005. Zur antiken Buchkultur allgemein: Blanck 1992; Mazal 1999.

4 Martial spricht von seinem Publikum fast ausschließlich als lector, der Begriff auditor fällt nur zweimal. Vgl. hierzu Howell 1980, 103f. zu Mart. 1,1,4.

5 Dies spiegelt sich unter anderem darin, dass seit Ovid die Figur des anonymen Lesers (e. g. am. 2,1 und trist. 3,1) die Szene betritt.

6 Mart. 1,1,4.

7 Etwa Mart. 4,55,27: delicatus; 10,59,5: gulosus. 
der geschmäcklerisch und wählerisch auf der Jagd nach literarischen Leckerbissen ist und alles, was seinem verwöhnten Gaumen nicht entspricht, auf der literarischen Speiseplatte liegen lässt, dem Literaturkritiker, der mit rhinozerosgleicher Nase Neuerscheinungen beschnüffelt, bis hin zum gelangweilten, bei der Lektüre gähnenden und nur auf das hoffentlich baldige Ende des Gedichtbuches schielenden Lesers. All diese Imaginationen sind natürlich auch dazu angetan, mit dem Leser in einen beständigen kommunikativen Prozess einzutreten, je neue Reize für seine Aufmerksamkeit zu setzen und bestimmte Rezeptionsmodi einzufordern. Selbstredend zielt diese Art des literarisch inszenierten Dialogs zwischen Autor und Leser auch darauf, eine Imagination von Nähe und Vertrautheit mit dem notwendigerweise unbekannten und durch eine kommunikative und räumliche Distanz vom Autor getrennten Publikum herzustellen.

\section{Der lector amicus und Imaginationen der Nähe}

In diesen Zusammenhang gehört auch die häufige Betonung einer affektiven Bindung zwischen Leser und Autor und die Apostrophierung des Lesers als amicus und amator von Text und Autor.

Bereits in den programmatischen Eröffnungsgedichten des ersten Epigrammbuches wird durch den Begriff des lector studiosus, mehr aber noch durch das in den Verben requiris, cupis und quaeris imaginierte Verlangen des Lesers dieses emotionale Nahverhältnis illustriert: ${ }^{8}$

\footnotetext{
Hic est quem legis ille, quem requiris, toto notus in orbe Martialis argutis epigrammaton libellis: cui, lector studiose, quod dedisti viventi decus atque sentienti, rari post cineres habent poetae.
}

Hier ist der, den du liest, nach dem du verlangst, Martial, in der ganzen Welt bekannt Durch die scharfzüngigen Büchlein seiner Epigramme: Den Ruhm, den du ihm, eifriger Leser, noch während er lebt und es empfinden kann, erwiesen hast, erreichen Dichter, selbst nachdem sie zu Asche geworden sind, selten nur. ${ }^{9}$

(Mart. 1,1)

8 Siehe hierzu Howell 1980 zur Stelle.

9 Übersetzung in Anlehnung an Barié/Schindler 2013. Dies gilt auch für die übrigen Übersetzungen aus Martials Epigrammen. 
Die Beziehung zum Leser wird als ein Verhältnis wechselseitiger Bedingtheit und Zuneigung inszeniert. Besonders deutlich kommt dies in Epigramm 5,16 zum Ausdruck, in dem die Wahl des Sujets und der Darstellungsmodus mit den Interessen der Leserschaft begründet, zugleich aber auch der Erfolg des Autors als Ausdruck einer engagierten Leserschaft, die in ein beinahe amouröses Verhältnis zum Dichter tritt, verstanden wird:

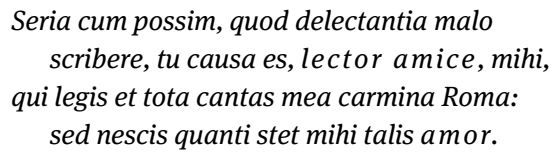

Zwar könnte ich auch Ernstes schreiben, doch dass ich Unterhaltsames vorziehe, dafür bist du mir Leser, mein Freund, der Grund, $\mathrm{du}$ der meine Gedichte liest und in ganz Rom singt.

Aber du weißt nicht wie teuer mich diese Liebe zu stehen kommt.

(Mart. 5,16)

Hinter dieser Verwendung des Begriffs amicus steht natürlich auch das System der als amicitia gefassten Patronage, die hier in innovativer Weise auf die Beziehung des Autors zum Lesepublikum übertragen wird. Dieser ausgesprochen komplexe Transfer kann im Rahmen der hier angestellten Überlegungen leider nicht weiter differenziert ausgeführt werden. ${ }^{10}$ Vielmehr soll hier auf die emotionale Steigerung, die sich in dem Begriff amor verbirgt, etwas näher eingegangen werden. Präsentiert sich hier Martial als opferbereiter amator seines Publikums, so wird andernorts durchaus auch die umgekehrte Perspektive - das Publikum als Liebhaber Martials und seiner Texte eingenommen. Hier sei nicht nur noch einmal auf die affektivische Aufladung der Leserreaktionen in den Eröffnungsgedichten verwiesen, sondern auch auf die explizite Formulierung des Sachverhalts in Epigramm 6,60 oder im Proömium zum neunten Buch: ${ }^{11}$

Laudat, amat, cantat nostros mea Roma libellos,

Mein Rom lobt, liebt, rezitiert meine Büchlein.

(Mart. 6,60,1)

Ille ego sum nulli nugarum laude secundus, quem non miraris, sed puto, lector, amas.

Ich bin derjenige, der mit seinen poetischen Nichtigkeiten hinter keinem an Ruhm zurücksteht, den $\mathrm{du}$, Leser, nicht bestaunst, sondern, glaube ich, liebst.

(Mart. 9 praef.)

10 Siehe hierzu etwa: Nauta 2002; Leberl 2004; Rühl 2006.

11 Hierzu auch unten S. 161f. 
Gerade in der Differenzierung zwischen einer auf bloßer Bewunderung und emotionaler Zuneigung beruhenden Leserreaktion soll die spezifische Qualität martialischer Epigramme als einer Dichtung ausgemacht werden, die in ganz besonderer Weise dazu angetan ist qua Text ein nachgerade intimes Verhältnis zwischen Leser und Autor herzustellen und damit die der literarischen Kommunikation mit einem anonymen Publikum strukturell gegebene Distanz zu überbrücken. Ein besonders illustratives Beispiel für die Permanenz der Gegenwart des Dichters im Buch in den unterschiedlichsten Lebenssituationen stellt etwa das Epigramm 12,1 dar:

Retia dum cessant latratoresque Molossi
et non invento silva quiescit apro,
otia, Prisce, brevi poteris donare libello.
Hora nec aestiva est nec tibi tota perit.

Während deine Netze und deine molossischen Kläffer nicht im Einsatz sind und Stille im Wald einkehrt, weil kein Eber sich zeigt, kannst du, Priscus, deine Muße meinem kleinen Büchlein zuwenden. Weder ist jetzt Sommerzeit, noch geht dir eine ganze Stunde verloren.

(Mart. 12,1)

Die Verknüpfung mit der Jagdsituation dient dabei nicht nur dazu die Qualität des Buches als Form der Freizeitbeschäftigung und der Unterhaltung auszuweisen, sondern verweist auch auf die enge Bindung zwischen Autor und Adressat, in diesem Fall seinem Patron Priscus, die sich hier aus der Tatsache ableitet, dass das Buch zu einem veritablen Begleiter des patronus wird.

Dabei bedient sich Martial nicht nur der terminologischen Propagierung mit Begriffen der amicitia und des amor. Vielmehr verwendet er ein ganzes Arsenal auch performativer Verfahren, um Vertrautheit und Nähe gleichsam in den Leseakt einzuschreiben.

Ein instruktives Beispiel für die performative Konstruktion von Nähe ist auch das metaleptische Heraustreten ${ }^{12}$ des Autors aus dem Text in zwei an eine weibliche Leserin gerichteten Epigrammen im 3. Buch. In Epigramm 3,68, das zugleich die Funktion eines Binnenproöms hat, wendet sich der Autor an seine als matrona gekennzeichnet Adressatin:

Huc est usque tibi scriptus, matrona, libellus.

Cui sint scripta, rogas, interiora? Mihi.

Gymnasium, thermae, stadium est hac parte: recede.

Exuimur: nudos parce videre viros.

Hinc iam deposito post vina rosasque pudore, quid dicat, nescit saucia Terpsichore:

schemate nec dubio, sed aperte nominat illam,

$12 \mathrm{Zu}$ Formen der Metalepse in antiker Literatur siehe Eisen/Möllendorff 2013. 
quam recipit sexto mense superba Venus,

custodem medio statuit quam vilicus horto,

opposita spectat quam proba virgo manu.

Si bene te novi, longum iam lassa libellum

ponebas, totum nunc studiosa leges.

Bis hierher ist das Büchlein für dich geschrieben, ehrbare Dame des Hauses.

Für wen dann die folgenden Seiten geschrieben sind, fragst du? Für mich!

Sporthalle, Thermen, Rennbahn, all das ist in diesem Teil: Entferne dich,

wir ziehen uns aus, erspar' es dir, nackte Männer anzusehen!

$\mathrm{Ab}$ jetzt, nach Wein und Rosen, verliert Terpsichore jede Scheu

Und weiß beschwipst nicht, was sie sagt.

Ganz unverblümt und offen nennt sie jetzt das Ding,

das Venus stolz im Monat Juni empfängt,

das als Wächter der Gutsverwalter mitten in den Garten stellt,

bei dessen Anblick ein anständiges Mädchen die Hand vor die Augen hält.

Kenn' ich dich recht, dann wolltest du das Büchlein wegen seiner Länge schon müde beiseite legen - und liest es jetzt eifrig zu Ende.

(Mart. 3,68)

Als verheiratete und ehrbare Frau solle also die Leserin nun die Lektüre des Buches beenden, da das weitere eine allzu freizügige und damit unschickliche Thematik habe. Martial eröffnet hier in Rede und Gegenrede einen launigen Dialog, in dem er die Leserin und sich selbst als Teil einer intimen Lesegemeinschaft inszeniert. Der Schluss des Epigramms ist dabei ein vorzüglicher Beleg für die oben angesprochene Leserlenkung und die Erzeugung von Aufmerksamkeit. Noch deutlicher ausgeprägt ist der metaleptische Charakter im korrespondierenden Epigramm 3,86:

\footnotetext{
Ne legeres partem lascivi, casta, libelli, praedixi et monui: tu tamen, ecce, legis.

Sed si Panniculum spectas et, casta, Latinum, non sunt haec mimis inprobiora - lege.
}

Einen Teil meines frivolen Büchleins nicht zu lesen, tugendhafte Dame Hab' ich dir vorher eingeschärft und dich gewarnt: doch siehe da, du liest es trotzdem! Nun, wenn du dir Panniculus und Latinus anschauen kannst, tugendhafte Dame, meine Verse sind nicht unanständiger als die Mimen - dann lies sie!

(Mart. 3,86)

So, als habe der Autor seiner Leserin bei der Lektüre beständig über die Schulter geschaut, ertappt er sie dabei, wie sie offenkundig entgegen dem Rat des Autors die Lektüre fortgesetzt hat. Die gleichsam physische Präsenz des Autors im Lesezimmer wird durch das deiktische ecce zusätzlich unterstrichen. Damit wird der prozessual verstandene Leseakt als Ort eines permanenten Dialogs zwischen Autor und Leser konstruiert, indem sich in der textuellen Vergegenwärtigung des Autors und in der Imagination des Lesers die enge Bindung und wechselseitige Wertschätzung mani- 
festiert, so dass man nachgerade von einer Kumpanei zwischen Leser und Autor sprechen könnte.

In diesen Kontext gehört natürlich auch die vielfältige Imagination von Rezeptionssituationen, von der zeitgenössischen Festkultur bis zum convivium, die darauf abzielt, einerseits eine Erfahrungs- und Erlebnisgemeinschaft zwischen Autor und Leser herzustellen und andererseits die Epigramme durch ihren Gegenstand und ihre Darstellungsweise als einer im Mittelpunkt der Lebenspraxis der Rezipienten stehende Form literarischer Unterhaltung auszuweisen und zu beglaubigen.

\section{Praktiken der Nähe oder im Körperkontakt mit dem Text}

Ein weiterer bislang nur wenig berücksichtigter Aspekt dieses Szenarios der Nähe stellen die durchaus nicht seltenen Verweise auf den physischen Kontakt zwischen Leser und Text als Ausdruck einer intensiven Lesepraxis dar. ${ }^{13}$ Damit wende ich mich zugleich dem eigentlichen Kern meiner Überlegungen zu, die darauf abzielen, die Handhabung unterschiedlicher textueller Formen in den Blick zu nehmen und u. a. auch die neue Form des handlichen Buches in Codexform auf die mit ihr verbundenen Wahrnehmungs- und Lesepraktiken zu befragen. ${ }^{14}$ Als Ausgangspunkt sollen abermals die beiden bereits angesprochenen Texte - das Epigramm 6,60 und die praefatio zu Buch 9 herangezogen werden. In beiden Texten manifestiert sich die Nähe von Leser und Autor ganz explizit in der physischen Handhabung des Buches. Ich zitiere zunächst das Epigramm aus der praefatio zu 9:

Maiores maiora sonent: mihi parva locuto

sufficit in vestras saepe redire manus.

13 Siehe dazu mit Blick auf die Elegie Ritter-Schmalz in diesem Band.

14 Ich verweise hier etwa auf die Arbeiten von Blanck 1992, 97-101 und Roberts/Skeat 1983, 24-34. Letztere vertreten entschieden die Meinung, bei den von Martial genannten Pergamentcodices handle es sich um eine Ausnahme, eine Art buchtechnischen Versuchsballon, dem kein übermäßiger Erfolg beschieden gewesen sei. Blanck macht zudem aus dem Schweigen des älteren Plinius ein Argument für die Ungebräuchlichkeit des Codex. Dennoch lassen sich meines Erachtens keine Aussagen darüber machen, ob es sich bei den hier angesprochenen Büchern tatsächlich um eine Novität handelt, noch lassen sich Rückschlüsse auf die tatsächliche Verbreitung solcher Bücher ziehen. Dies umso mehr, als es sich allem Dafürhalten nach um eine spezielle funktionsgebundene Art der literarischen Publikation handelte, deren Erzeugnisse vorwiegend als bequem transportfähige Reiseliteratur Verwendung finden sollten. Die Bedeutung der Materialität des Buches nimmt auch Roman 2001 in den Blick, fokussiert aber in erster Linie auf die Bedeutung des Buches als Teil einer auf vordergründige Selbstherabsetzung der Dichterpersona im Unterschied zu augusteischen Selbstdarstellungspraktiken. 
Größere mögen von Größerem tönen: für mich der nur Unbedeutendes geäußert hat, genügt es, wenn ihr mich oft wieder in Eure Hände nehmt.

(Mart. 9 praef.)

Die Vorstellung vom Buch in den Händen des Lesers und die damit verbundene Apostrophierung eines physischen Aspektes des Lesevorgangs mag auf den ersten Blick als bloß oberflächliche Lektüremetapher erscheinen. Bei einem genaueren Blick zeigt sich aber, dass damit durchaus auch die Vorstellung einer sich in diesem physischen Vorgang manifestierenden Vertrautheit von Autor und Leser im Medium des Textes mitgedacht wird. Dies zeigt sich zumal in einer durchaus gängigen Junktur, die die Hände des Lesers mit der Verwahrung des Buches im Gewandbausch verknüpft. Betrachten wir in diesem Zusammenhang noch einmal Epigramm 6,60. Dort heißt es wie folgt:

Laudat, amat, cantat nostros mea Roma libellos, meque sinus omnes, me manus omnis habet.

Ecce rubet quidam, pallet, stupet, oscitat, odit. Hoc volo: nunc nobis carmina nostra placent.

Mein Rom lobt, liebt, rezitiert meine Büchlein, mich birgt jeder Gewandbausch, mich hält jede Hand.

Sieh, manch einer wird rot, erbleicht, stutzt, sperrt den Mund auf, empört sich.

Und genau das will ich: jetzt gefallen mir meine Gedichte.

(Mart. 6,60)

In diesem Text wird mit allem Nachdruck jener Mehrwert markiert, der mir für die Rede vom Buch in der Hand signifikant zu sein scheint. Ganz offenkundig geht es hier nicht nur um das factum brutum der enormen Verbreitung von Martials Texten und der Vielschichtigkeit seines Publikums, sondern auch um die besondere Intensität dieser Beziehung. Die libelli werden nicht nur von allen möglichen Lesern in die Hand genommen und zur Lektüre hervorgeholt, sondern sind auch beständige Begleiter der Leser. Gerade dem Verweis auf die Verwahrung des Buches im Gewandbausch eignet hier eine besondere Dimension von Intimität. Zwar erscheinen Gewandbäusche bei Martial in durchaus unterschiedlichster Form als Aufbewahrungs- und Transportort für ausgefallene Zähne, ${ }^{15}$ erbeutete Leckerbissen des Mahlzeitenjägers, ${ }^{16}$ üppige Barschaft eines Geizkragens, ${ }^{17}$ Spezereien, die Zoilus vom Scheiterhaufen eines Verstorbenen an sich gebracht hat, ${ }^{18}$ oder gar für die Gebeine eines Verstorbenen, ${ }^{19}$ doch

15 Mart. 8,57.

16 Mart. 7,20.

17 Mart. 4,51.

18 Mart.11,54.

19 Mart. 9,30. 
lässt sich durchaus feststellen dass bereits selbst in diesen eher skurrilen Fällen der Gewandbausch nicht nur als bequeme Transportmöglichkeit erscheint, sondern nicht zuletzt mit Gegenständen in Verbindung gebracht wird, die dem Träger des Gewandes lieb und teuer sind oder zumindest seine besondere Aufmerksamkeit genießen. Dies gilt nun auch und erst recht für Bücher, für die der Gewandbausch nicht nur temporäres Transportmittel, sondern dauerhafter Aufenthaltsort, ja sogar geschützter Zufluchtsort wird. Als programmatisch für das Verhältnis von patronus und poeta cliens wird man hier das an Faustinus gerichtete Epigramm 3,2 betrachten dürfen:

\author{
Cuius vis fieri, libelle, munus? \\ Festina tibi vindicem parare, \\ ne nigram cito raptus in culinam \\ cordylas madida tegas papyro \\ vel turis piperisve sis cucullus. \\ Faustini fugis in sinum? Sapisti. \\ Cedro nunc licet ambules perunctus \\ et frontis gemino decens honore \\ pictis luxurieris umbilicis, \\ et te purpura delicata velet, \\ et cocco rubeat superbus index. \\ Illo vindice nec Probum timeto.
}

\begin{abstract}
Wem willst du zum Geschenk werden, mein Büchlein?
Beeile dich, dir einen Beschützer zu besorgen,

damit man dich nicht alsbald zur rauchgeschwärzten Küche entführt

und du mit feuchtem Papyrus Thunfische zudeckst

oder als Tüte für Weihrauch oder Pfeffer Verwendung findest!

Flüchtest du in den Gewandbausch von Faustinus? Du hast Geschmack.

Mit Zedernöl gesalbt, kannst du so deinen Weg gehen

und, beiderseits an den Außenrändern dekorativ gestaltet,

mit den bemalten Buchrollenknäufen prahlen;

auch soll dich feiner Purpur umhüllen

und stolz der scharlachrote Titel leuchten.

Bei so einem Beschützer brauchst du nicht einmal den Probus zu fürchten.

(Mart. 3,2)
\end{abstract}

Der deutlich an Catull ${ }^{20}$ angelehnte Text, der die Bedeutung eines patronus für die Buchproduktion illustriert und in witziger Weise das Spannungsverhältnis von literarischer Qualität und äußerem Erscheinungsbild des Buches thematisiert, fasst die Schutzfunktion des patronus in das suggestive Bild des Gewandbausches, in dem das Büchlein Zuflucht finden kann. So sehr hier auch mit einer metaphorischen Verwendung des Begriffs zu rechnen ist, belegt der Text gleichwohl dass mit sinus eine besondere emotionale Aufmerksamkeit, ja beschützende Fürsorge verbunden ist.

20 Zur Catullrezeption bei Martial etwa Swann 1994; Lorenz 2007. 
Auf einer deutlich weniger metaphorischen Ebene bewegt sich Epigramm 2,6, in dem uns Martial einen Leser vorstellt, der zwar Martials Epigrammbücher nicht zu goutieren weiß, aber einzelne Epigramme sofort nach dem Vortrag des Dichters, auf kostbaren Papyrus geschrieben, im Gewandbausch allerorten vom Theater bis zum convivium bei sich trägt. Schwingt hier natürlich die Konnotation des Plagiats und des möglichen Vortrags von Martials Gedichten als Eigenproduktion mit, ${ }^{21}$ ist der Sachverhalt in Epigramm 3,5 dagegen in diesem Sinne völlig unverdächtig:

Vis commendari sine me cursurus in urbem, parve liber, multis, an satis unus erit?

Unus erit, mihi crede, satis, cui non eris hospes, Iulius, assiduum nomen in ore meo.

Protinus hunc primae quaeres in limine Tectae: Quos tenuit Daphnis, nunc tenet ille lares.

Est illi coniunx, quae te manibusque sinuque excipiet, tu vel pulverulentus eas.

Hos tu seu pariter sive hanc illumve priorem videris, hoc dices ,Marcus havere iubet", et satis est: alios commendet epistula: peccat qui commendandum se putat esse suis.

Willst du mein Büchlein, wenn du jetzt ohne mich nach Rom eilst, daß ich dich vielen empfehle, oder ist dir einer genug? Einer, glaub mir, genügt, und bei ihm wirst du kein Fremder sein: Julius, sein Name ist stets in meinem Mund.

Gleich zu Beginn der Säulenhallenstraße mußt du ihn suchen; Er besitzt jetzt das Haus, das früher Daphnis besaß, er hat eine Frau, die dich mit ihren Händen und an ihrem Herzen Aufnimmt, auch wenn du staubbedeckt ankommst. Siehst du sie beide zusammen, siehst du zuerst sie oder ihn, dann brauchst du nur zu sagen: „Marcus lässt grüßen.“ Das genügt, andere mag ein Brief empfehlen: Falsch handelt, wer meint, sich den Seinen empfehlen zu müssen.

(Mart. 3,5)

Dieser Text thematisiert in vielfältiger Weise die hier verhandelte Inszenierung von Nähe und Vertrautheit. Dies reicht vom Zwiegespräch des Buches mit den Adressaten bis hin zur Apostrophierung der hier vorgestellten Leser als sui, als vertraute Freunde des Autors. In unserem Zusammenhang ist aber v. a. der Empfang des Buches durch die Frau des Hauses von Interesse. Hier werden die Begriffe manus und sinus in ein besonderes Verhältnis zueinander gerückt. Das Buch in die Hand zu nehmen verweist dabei auf die gängige Rezeption eines Textes, während die Aufnahme im sinus der Hausherrin auf eine spezifisch emotionale Verbundenheit der Leser mit dem Text und

21 Zum Thema der Plagiierung bei Martial Fitzgerald 2007, 93-97. 
seinem Autor zielt. Man könnte nachgerade von einer erotischen Aufladung der Begegnung des Buches mit dem Leser sprechen, eine Dimension, die sich auch durch Verwendung des Begriff sinus im Kontext erotischen Begehrens wie etwa in Martial 12,49 erhärten ließe:

Crinitae Line paedagoge turbae, rerum quem dominum vocat suarum et credit cuï Postumilla dives gemmas, aurea, vina, concubinos: Sic te perpetua fide probatum nulli non tua praeferat patrona: Succurras misero, precor, furori et serves aliquando neglegenter illos qui male cor meum perurunt, quos et noctibus et diebus opto in nostro cupidus sinu videre, formosos, niveos, pares, gemellos, grandes, non pueros, sed uniones.

Du Aufseher der langhaarigen Schar, Linus, den als ihres Vermögens Herrn die reiche Postumilla bezeichnet und dem sie anvertraut Juwelen, Goldstücke, Weine und Beischläfer: So wahr dich, den in dauerhafter Treue bewährten, deine Patronin jedem anderen vorziehen möge, so bestimmt eile zu Hilfe, ich bitte darum, meinem elenden Wahn, und pass einmal wenigstens nachlässig auf die auf, die mein Herz so arg entflammen, die ich Tag und Nacht begierig an meinem Busen zu sehen wünsche, jene wohlgestalteten, schneeweißen, ebenmäßigen, zwillingsgleichen, stattlichen - Knaben nicht, nein Perlen.

(Mart. 12,49)

Vor diesem Hintergrund gewinnen nun auch die Aussagen über die innovative Buchform des Codex eine neue Dimension. Hierzu sei zunächst einmal Epigramm 1,2 in den Blick genommen, das Teil einer ganzen Serie von poetologischen Texten ist, mit denen Martial im ersten Epigrammbuch den programmatischen Horizont seines Werkes unter den Aspekten Autor, Text und Leserschaft und damit aller relevanten Aspekte literarischer Kommunikation absteckt:

Qui tecum cupis esse meos ubicumque libellos et comites longae quaeris habere viae, hos eme, quos artat brevibus membrana tabellis: Scrinia da magnis, me manus una capit.

Ne tamen ignores ubi sim venalis, et erres urbe vagus tota, me duce certus eris: 
Libertum docti Lucensis quaere Secundum
limina post Pacis Palladiumque forum.

Wenn du meine Büchlein überall bei dir haben willst Und sie dir als Begleiter für eine lange Reise wünschst, dann kaufe diese hier: Das Pergamentformat reduziert sie auf eine knappe Zahl von Blättern. Buchrollenbehälter verwende für die großen Werke, mich kann man mit einer Hand fassen. Damit du aber genau weißt, wo ich zu kaufen bin, und nicht ziellos Herumirrst in der ganzen Stadt, wirst du an mir einen zuverlässigen Führer haben: Suche Secundus auf, den Freigelassenen des gelehrten Lucensis, hinter der Schwelle des Pax-Tempels und dem Forum der Pallas.

(Mart. 1,2)

Die hier im Blick auf die neue Buchform propagierte Lesepraxis kann man durchaus als eine durch das Miniaturformat bedingte Intensivierung der bislang behandelten Praktiken verstehen. Dies gilt sowohl für den Umfang der mitgeführten Texte - explizit wird ja der Plural libelli, das heißt wohl mindesten die Bücher 1 und 2 der Epigramme Martial verwendet - und auch für die Dauer der Nutzung der Texte, die hier in der Metaphorik der Reise greifbar wird. Longa via und ubicumque imaginieren eine zeitlich wie räumlich uneingeschränkte Lektürebeziehung zu Text und Autor, die wie so oft bei Martial sich wechselseitig vertreten können. Dabei geht es eben nicht nur um die schlichte Transportabilität des Textes, ein Aspekt, der v. a. unter buchtechnischen Rücksichten bei der Diskussion dieses Epigramms unter dem Stichwort Reiseliteratur immer geltend gemacht wurde, sondern auch um eine vertiefte Präsenz von Autor und Buch. Gerade die Vorstellung vom Buch als ubiquitär verfügbarer Reisebegleiter und die damit verbundene Personifizierung des Textes als eines vertrauten Freundes, der den Leser niemals und nirgends im Stich lässt, markiert diese neue Qualität von der Intimität des Lektüreaktes. Die Hand des Lesers ist dabei nicht nur Leseinstrument, sondern in gewisser Weise Ort der physischen Begegnung zwischen Leser und Autor. Ein Blick auf ein Epigramm aus der Reihe der in den Apophoreta versammelten Buchgeschenkepigramme ${ }^{22}$ auf eine Ciceroausgabe in Codexform mag dies noch verdeutlichen:

\footnotetext{
Si comes ista tibi fuerit membrana, putato

carpere te longas cum Cicerone vias.
}

Wenn dies Pergament dein Begleiter ist, dann stelle dir vor, daß du eine weite Reise mit Cicero machst.

(Mart. 14,188)

$22 \mathrm{Zu}$ den literarisch konzeptuellen Implikationen dieser Epigramme Neger 2012, 22-48; Mindt 2013, 25-29. Zu Aspekten von Materialität und Handhabbarkeit Borgo 2004. 
Natürlich wird man auch dieses Epigramm zunächst einmal als ein Zeugnis für die Lesepraxis auf Reisen lesen können, aber in der Figuration des comes lässt sich natürlich auch der oben beschriebene Mehrwert greifen. Der Leseakt ist eben nicht bloße Lektüre, sondern konstituiert eine unmittelbare Erfahrung des Autors und wird zum Ort seiner Vergegenwärtigung, die in diesem Falle sogar die Zeiten zu überspringen vermag. Martials Insistieren auf dieser Imagination der Nähe projiziert in gewisser Weise die Erfahrung der Distanz, die durch einen in gewisser Weise entpersonalisierten Buchmarkt erzeugt wird, damit generell auf alle Formen von Lektüre.

\section{Buch und Bild. Strategien der Vergegenwärtigung}

Eine weitere mit der Codexform verbundene Neuerung kann durchaus als Verstärkung dieser Form der Vergegenwärtigung verstanden werden. Offenbar nutzte man nämlich das neue Buchmedium auch dazu, die Texte mit einem Autorenporträt zu versehen. ${ }^{23}$ So etwa bei der ebenfalls im o. g. Kontext der Apophoreta behandelten Vergilausgabe:

\section{Quam brevis inmensum cepit membrana Maronem! Ipsius vultus prima tabella gerit.}

Welch kleines Pergament fast den gewaltigen Maro! Ein Porträt von ihm selbst bringt das erste Blatt.

(Mart. 14,186)

Diese hier scheinbar eher beiläufige Bemerkung zur aufwendigen Gestaltung von Pergamentcodices gewinnt im Lichte einiger Martialepigramme, die das Verhältnis von Autorenporträt und Text ausführlicher reflektieren, eine für unseren Kontext ausgesprochen einschlägige Dimension.

Dazu möchte ich zunächst noch einmal auf die bereits thematisierte praefatio zu Buch 9 eingehen. ${ }^{24}$ Die praefatio, die aus einer kurzen Prosaepistel und einem von Martial explizit als extra ordinem paginarum bezeichneten Epigramm besteht, imaginiert eine komplexe Kommunikationssituation. ${ }^{25}$ Der kurze Brief richtet sich an Mar-

23 Die systematische Bilddarstellung bedeutender auch literarischer Persönlichkeiten im Medium des Buches lässt sich vermutlich auf die Imagines des Varro, ein biographisches Bildlexikon mit 700 Porträts zurückführen. Möglicherweise hatte dies auch unmittelbar Auswirkungen auf die Präsentation von Autoren in öffentlichen Bibliotheken. Die Publikation der Imagines liegt geschieht jedenfalls im gleichen zeitlichen Kontext wie der erste große Bau einer zweisprachigen Bibliothek in Rom durch Asinius Pollio, für die die Ausstellung von Autorenporträts explizit bezeugt ist.

24 Siehe auch oben S. $165 f$.

25 Siehe hierzu Johannsen 2006, 98-106. Die Rolle des Bildmediums und seine Funktion wird hier allerdings nicht hinreichend berücksichtigt. 
tials Freund Toranius und erläutert diesem, wer der Adressat des Epigramms ist. Bei diesem handelt sich um Stertinius Avitus Suffektkonsul des Jahres 92, einen Dichter, Freund und Patron Martials. Dieser hatte in seiner Privatbibliothek, die nach gängiger Praxis auch Porträtbüsten von Autoren enthielt, ${ }^{26}$ auch für Martial eine solche Büste aufstellen lassen. Martial hatte nun als subscriptio dieses Bildnisses das im folgenden zitierte Epigramm verfasst, das einerseits sein Verhältnis zu Avitus, andererseits, ganz im Stile vergleichbarer Epigramme in öffentlichen Bibliotheken, ein literarisches Porträt des Autors liefert. Das Zusammenwirken von durch den patronus in Auftrag gegebenem Bildwerk und Epigramm konstituiert eine Form des wechselseitigen Kompliments und der wechselseitigen Bespiegelung von patronus und Dichter, wie sie für die Patronageliteratur der Zeit, insbesondere auch bei Statius und in gewisser Weise auch bei Plinius dem Jüngeren, ${ }^{27}$ keineswegs ungewöhnlich ist. Die statuarische Würdigung Martials manifestiert zugleich die enge Beziehung, die zwischen Avitus und dem Epigrammatiker besteht, und kann und soll nicht zuletzt als Ausdruck enger Vertrautheit und als Imagination der Nähe verstanden werden. Gerade dieser Aspekt wird nun in einer Formulierung greifbar, mit der Martial gegenüber Avitus die Wirkung des eigenen Epigramms in dem oben bezeichneten Kontext beschreibt. In Vers 3 des Epigramms heißt es nämlich: hoc tibi sub nostra breve carmen imagine vivat - „dies kurze Gedicht soll für dich unter meinem Bilde leben“. Vivat ist auf den ersten Blick ein äußerst ungewöhnlicher Ausdruck, der durchaus interpretationsbedürftig ist. Natürlich ist die Rede von der Lebendigkeit eines Kunstwerkes im Kontext der Bildkunst eine gängige Metapher, um die Qualität der Darstellung als lebensecht und gelungen $\mathrm{zu}$ bezeichnen. Es wäre hier etwa auf die zahlreichen Epigramme auf die myronische Kuh zu verweisen, in der das Vexierspiel zwischen Bildnis und Wirklichkeit vielfältig ausgebeutet wird. ${ }^{28}$ Martial transponiert nun die dem Bildmedium eigene Anschaulichkeit und Vergegenwärtigungsleistung auf das Epigramm und damit den literarischen Text. Er operiert dabei mit einem Topos, der uns von den fiktiven Grabepigrammen hellenistischer Couleur bekannt ist, in der das tote Monument im performativen Sprechakt Lebendigkeit erlangt. ${ }^{29}$

Erst im Zusammenspiel mit dem Gedicht gewinnt das Porträt, das durch das Epigramm zum Sprechen gebracht wird, also eine Qualität, die man als Erzeugung einer sich im Leseakt permanent herstellenden Vergegenwärtigung des Dichters beschreiben könnte und die Martial auch aus der Ferne zum Hausgenossen des Avitus macht.

Damit greifen wir sicher auch eine wesentliche Funktion von Porträtbildnissen in Büchern, deren Verhältnis Martial auch in 7,84 reflektiert, einem Gedicht, in dem er abermals unter Bezugnahme auf die Erstellung eines Autorenprorträts, das er als atmendes und damit lebendiges Bildnis beschreibt, auf den Text als eigentlichen Ort

$26 \mathrm{Zu}$ Porträtbüsten in Privatbibliotheken Plin. nat. 35,9-11.

27 Zur Reziprozität des Kompliments z. B. Plin. epist. 1,17 oder Stat. silv. 1,3. Siehe hierzu Krasser 1993.

28 Siehe hierzu Squire 2010.

29 Siehe hierzu etwa Männlein-Robert 2007, 154-186. 
der bildhaften Imagination des Dichters verweist. Certior in nostro carmine vultus erit heißt es dort (Mart. 7,84,6) und in ähnlicher Formulierung wie in praefatio 9: vivet, Apelleum cum morietur opus (Mart. 7,84,9).

Martial bedient sich hier natürlich des diskursiven Arsenals des Paragone der Künste und hebt damit auf die den Bildwerken überlegene Qualität seiner Dichtung ab. Doch zugleich wird durch die Kommunikationssituation des Textes, der sich an Caecilius Secundus, einen bei einem Militärkommando an der Donau weilenden patronus, richtet, der offenkundig neben der Übersendung der Texte um ein gemaltes Autorenporträt des Dichters gebeten hatte, eine Lesepraxis sichtbar, die den Text auch und nicht zuletzt als einen Akt der Imagination des Autors, seiner Vergegenwärtigung und damit als nachgerade physischen Begegnungsort zwischen Leser und Dichter versteht.

Stellt man diese Kommunikations- und Wahrnehmungspraxis in Rechnung, so hätte sich unser eingangs zitierte Gaditaner vielleicht auch seine lange Reise ersparen können.

\section{Literaturverzeichnis}

Barié, Paul/Schindler, Winfried (2013³), M. Valerius Martialis. Epigramme. Lateinisch - deutsch, hg. und übers. von P. B. und W. S., Berlin.

Binder, Gerhard (1995), „Öffentliche Autorenlesungen. Zur Kommunikation zwischen römischen Autoren und ihrem Publikum“ in: Gerhard Binder u. Konrad Ehlich (Hgg.), Stätten und Formen der Kommunikation im Altertum IV (Bochumer Altertumswissenschaftliches Colloquium 23), Trier.

Blanck, Horst (1992), Das Buch in der Antike, München.

Borgo, Antonella (2004), „Un libro in dono. Su alcuni epigrammi degli Apophoreta di Marziale“, in: Giovanni Indelli, Giuliana Leone u. Francesco Longo Auricchio (Hgg.), Mathesis e Mneme. Studi in memoria di Marcello Gigante (Pubblicazioni del Dipartimento di Filologia Classica dell'Università degli Studi di Napoli 11), Neapel, 161-171.

Citroni, Mario (1975), M. Valerii Martialis epigrammaton liber primus, hg. von M. C., Florenz.

Citroni, Mario (1988), „Publicazione e dediche dei libri in Marziale“, in: Maia 40, 3-39.

Eisen, Ute E./von Möllendorff, Peter (Hgg.) (2013), Über die Grenze. Metalepse in Text- und Bildmedien des Altertums (Narratologia 39), Berlin.

Fantham, Elaine (1998), Literarisches Leben im antiken Rom. Sozialgeschichte der römischen Literatur von Cicero bis Apuleius, übers. von Theodor Heinze, Stuttgart/Weimar.

Howell, Peter (1980), A Commentary on Book One of the Epigrams of Martial, London.

Johannsen, Nina (2006), Dichter über ihre Gedichte. Die Prosavorreden in den Epigrammaton libri Martials und in den Silvae des Statius, Hypomnemata 166, Göttingen.

Kleberg, Tönnes (1967), Buchhandel und Verlagswesen in der Antike, Darmstadt.

Krasser, Helmut (1993), „Claros colere viros oder über engagierte Bewunderung. Zum Selbstverständnis des jüngeren Plinius“, in: Philologus 137, 62-71.

Krasser, Helmut (1999), „Lesekultur als Voraussetzung für die Rezeption von Geschichtsschreibung in der Hohen Kaiserzeit“, in: Martin Zimmermann (Hg.), Geschichtsschreibung und politischer Wandel im 3. Jh. n. Chr. (Historia Einzelschriften 127), Stuttgart, 57-69. 
Krasser, Helmut (2005), „Universalisierung und Identitätskonstruktion. Formen und Funktionen der Wissenskodifikation im kaiserzeitlichen Rom“, in: Günter Oesterle (Hg.), Erinnerung, Gedächtnis, Wissen. Studien zur kulturwissenschaftlichen Gedächtnisforschung (Formen der Erinnerung 26), Göttingen, 357-376.

Leberl, Jens (2004), Domitian und die Dichter. Poesie als Medium der Herrschaftsdarstellung (Hypomnemata 154), Göttingen.

Lorenz, Sven (2007), „Catullus and Martial“, in: Marilyn B. Skinner (Hg.), A Companion to Catullus, Malden (MA), 418-438.

Männlein-Robert, Irmgard (2007), Stimme, Schrift und Bild. Zum Verhältnis der Künste in der hellenistischen Dichtung (Bibliothek der Altertumswissenschaften. N. F. 2. Reihe, 119) Heidelberg.

Mazal, Otto (1999), Griechisch-römische Antike (Geschichte der Buchkultur 1), Graz.

Mindt, Nina, (2013), Martials, epigrammatischer Kanon ‘ (Zetemata 146), München.

Nauta, Ruurd Robijn (2002), Poetry for Patrons. Literary Communication in the Age of Domitian (Mnemosyne. Supplementum 206), Leiden/Boston.

Neger, Margot (2012), Martials Dichtergedichte. Das Epigramm als Medium der poetischen Selbstreflexion (Classica Monacensia 44), München.

Roman, Luke (2001), „The Representation of Literary Materiality in Martial's Epigrams“, in: The Journal of Roman Studies 91, 113-145.

Rühl, Meike (2006), Literatur gewordener Augenblick. Die Silven des Statius im Kontext literarischer und sozialer Bedingungen von Dichtung (Untersuchungen zur antiken Literatur und Geschichte 81), Berlin.

Squire, Michael (2010), „Making Myron's Cow Moo? Ecphrastic Epigram and the Poetics of Simulation“, in: American Journal of Philology 131, 589-634.

Swann, Bruce W. (1994), Martial's Catullus. The Reception of an Epigrammatic Rival (Spudasmata 54), Hildesheim.

Vössing, Konrad (1997) „Bibliothekswesen B: Griechenland, Rom, christliche Bibliotheken“, in: Der Neue Pauly 2, 640-647. 